\title{
Notes on lifting of Bertrand curve on tangent space $T R^{3}$
}

\author{
HAŞIM ÇAYIR ${ }^{\dagger}$ AND FIDAN JABRAILZADE
}

\author{
Date of Receiving : $\quad 02.02 .2018$ \\ Date of Revision : $\quad 20.12 .2018$ \\ Date of Acceptance : 23.12 .2018
}

\begin{abstract}
In this paper, firstly, we define the Bertrand curve of any curve with respect to the vertical, complete and horizontal lifts on space $R^{3}$ to its tangent space $T R^{3}=R^{6}$. Secondly, we examine the Frenet-Serret aparatus $\left\{T^{*}(s), N^{*}(s), B^{*}(s), \kappa^{*}, \tau^{*}\right\}$ and the Darboux vector $W^{*}$ of the Bertrand curve $\alpha^{*}$ according to the vertical, complete and horizontal lifts on $T R^{3}$ by depend on the lifting of Frenet-Serret aparatus $\{T(s), N(s), B(s), \kappa, \tau\}$ of the first curve $\alpha$ on space $R^{3}$. As a result of this transformation on space $R^{3}$ to its tangent space $T R^{3}$, we can speak about the features of Bertrand curve of any curve on space $T R^{3}$ by looking at the characteristics of the first curve.
\end{abstract}

\section{Introduction}

In differentiable gometry, lift method has an important role. Because, it is possible to generalize to differentiable structures on any space (resp. manifold) to extended spaces (resp. extended manifolds) using lift function $[4,5,6,7,8,10]$. Thus, it may be extended the following theorem given on space $R^{3}$ to its tangent space $T R^{3}$. It is well known that many studies related to the differential geometry of curves have been made. Especially, by establishing relations between the Frenet Frames in mutual points of two curves several theories have been obtained. The best known of the Bertrand curves discovered by J. Bertrand in 1850 are one of the important and interesting topics of classical special curve theory. A Bertrand curve is defined as a special curve which shares its principal normals with another special curve, called Bertrand mate or Bertrant curve partner. If

$$
\alpha^{*}=\alpha+\lambda N, \lambda=\text { constant }
$$

then $\left(\alpha, \alpha^{*}\right)$ are called Bertrand curve pair. If $\alpha$ and $\alpha^{*}$ Bertrand curves pair, then $\left\langle T, T^{*}\right\rangle=\cos \theta=$ constant $[2,3]$. The definition of $n$-dimensional Bertrand curves

2010 Mathematics Subject Classification. 28A51,53A04,57R25.

Key words and phrases. Vector fields; Bertrand curve; vertical lift; complete lift; horizontal lift; tangent space.

The authors are thankful to the referee(s) for their valuable time and suggestions towards the improvement of this paper.

Communicated by: A. Salimov

${ }^{\dagger}$ Corresponding author. 